\title{
Low validity of the Sensewear Pro3 activity monitor compared to indirect calorimetry during simulated free living in patients with osteoarthritis of the hip
}

\author{
Andreas Hermann ${ }^{1,2,3,7^{*}}$, Mathias Ried-Larsen ${ }^{4}$, Andreas Kryger Jensen ${ }^{5}$, René Holst ${ }^{6}$, Lars Bo Andersen ${ }^{4}$, \\ Søren Overgaard ${ }^{1,2}$ and Anders Holsgaard-Larsen ${ }^{1}$
}

\begin{abstract}
Background: To validate physical activity estimates by the Sensewear Pro3 activity monitor compared with indirect calorimetry during simulated free living in patients diagnosed with osteoarthritis of the hip pre or post total hip arthroplasty.

Methods: Twenty patients diagnosed with hip osteoarthritis (10 pre- and 10 post total hip arthroplasty; $40 \%$ female; age: $63.3 \pm 9.0 ; \mathrm{BMl}: 23.7 \pm 3.7$ ). All patients completed a 2 hour protocol of simulated free living with 8 different typical physical activity types. Energy consumption ( $\mathrm{kcal} / \mathrm{min}$ ) was estimated by the Sense Wear pro3 Armband activity monitor and validated against indirect calorimetry (criterion method) by means of a portable unit $\left(\right.$ Cosmed $K 4 \mathrm{~b}^{2}$ ). Bias and variance was analyzed using functional ANOVA.

Results: Mean bias during all activities was $1.5 \mathrm{Kcal} / \mathrm{min} 95 \% \mathrm{Cl}[1.3 ; 1.8]$ corresponding to $72 \%$ (overestimation). Normal gait speed showed an overestimation of $2.8 \mathrm{Kcal} / \mathrm{min}, 95 \% \mathrm{Cl}$ [2.3; 3.3] (93\%) while an underestimation of $-1.1 \mathrm{Kcal} / \mathrm{min}, 95 \% \mathrm{Cl}[-1.8 ;-0.3]$ (-25\%) was recorded during stair climb. Activities dominated by upper body movements showed large overestimation with $4.37 \mathrm{Kcal} / \mathrm{min}, 95 \% \mathrm{Cl}$ [3.8; 5.1] (170\%) being recorded during gardening. Both bias and variance appeared to be dependent on activity type.

Conclusion: The activity monitor generally overestimated the energy consumption during common activities of low to medium intensity in the patient group. The size and direction of the bias was highly dependent on the activity type which indicates the activity monitor is of limited value in patients with hip osteoarthritis and that the results do not express the real energy expenditure.
\end{abstract}

\section{Background}

Patients with osteoarthritis $(\mathrm{OA})$ of the hip have excess all cause mortality including increased mortality related to cardiovascular disease which has been associated with reduced patient reported physical activity (PA) [1]. Studies indicate that a majority of patients with lower extremity OA may not meet general recommendations regarding PA [2]. However, as the same is evident for the elderly

\footnotetext{
* Correspondence: ahermann@dadlnet.dk

'Orthopedic Research Unit, Department of Orthopaedic Surgery and

Traumatology, Odense University Hospital, Odense, Denmark

${ }^{2}$ Institute of Clinical Research, University of Southern Denmark, Odense,

Denmark

Full list of author information is available at the end of the article
}

population in general [3] it is still uncertain to which extend the functional impairment and pain present in symptomatic hip OA affects the actual PA compared with the general population. Studies of the objectively measured PA in patients with hip OA and total hip arthroplasty (THA) are limited especially regarding comparison with healthy controls [4-6] and their results are restricted by the general lack of validation studies of the used data collecting tools applied in patients with degenerative joint disease. Thus, establishing knowledge of the validity of objective measured PA in hip osteoarthritis patients is of importance for future research.

\section{() Biomed Central}

(c) 2014 Hermann et al.; licensee BioMed Central Ltd. This is an Open Access article distributed under the terms of the Creative Commons Attribution License (http://creativecommons.org/licenses/by/2.0), which permits unrestricted use, distribution, and reproduction in any medium, provided the original work is properly credited. 
PA is defined as any bodily movements produced by skeletal muscles that require energy expenditure [7]. Energy expenditure during PA is commonly investigated by indirect calorimetry which requires either isolation of individuals in closed spaces or portable apparatus for gas analysis of air exchange $[8,9]$. These methods are often referred to as criterion methods [10] but their application is limited to small laboratory settings $[8,9]$. In free living small bodyworn multisensory activity monitors based on accelerometry can be used as a feasible surrogate measure of energy expenditure during PA [11-13]. In clinical studies such activity monitors are applied due to their objectivity compared to self-reported physical activity questionnaires $[5,14]$ and they may become a tool in future etiological and prognostic studies in patients with lower extremity osteoarthritis [15]. However, in hip OA patients altered movement patterns may occur [16,17] and functional impairment and pain may affect the speed of exercises both potentially affecting estimations of energy expenditure based on accelerometry.

In the current study the Sensewear pro3 (SWA) activity monitor armband was validated. The SWA is a small multisensory activity monitor that combines accelerometry with various physiological data (see Method; Equipment). The monitor requires minimal instruction in use which suits the application in free living studies. The outcome in terms of energy expenditure is readily comparable with recommendations for PA (e.g. The American College of Sports Medicine [18]). Recently, the SWA has been used in a various clinical studies of actual PA in different patient groups [14,19-23] and in OA patients the monitor has been applied in a comparable study of PA between patients with hip and knee OA and healthy controls [5]. However, like other activity monitors the validity in this patient group is unknown. Varying degrees of bias has been observed when validated in healthy older adults $[11,24]$, obese adults [25] and in various patient groups including patients with rheumatoid arthritis [13,26-28]. In healthy adults the SWA been reported reliable [25,29] and valid for estimation of cumulated daily energy expenditure $[12,30]$. However, limitations regarding validity has been reported during various activity types [31-34].

The purpose of this study was to investigate the validity of the SWA activity monitor in patients with hip osteoarthritis during a simulated free living protocol according to the following 3 proprieties: i) Bias between activity monitor estimates and indirect calorimetry (criterion method), ii) correlation between methods and iii) difference in variance [35].

\section{Method}

\section{Participants}

A convenience sample of 20 patients (10 of preoperative stage, 10 of postoperative stage) diagnosed with hip OA
(Gender: 40\% female, Age: $63.3 \pm 9.2$ years, BMI: $23.7 \pm 3.8$ ) treated with THA or scheduled for THA at the Department Orthopaedic Surgery and Traumatology, Odense University Hospital (12 males, 8 females), were included.

\section{Inclusion/exclusion}

Inclusion criteria for the preoperative group: Diagnosed primary OA of the hip and scheduled for surgery (THA).

Inclusion criteria for the postoperative group: Diagnosed primary OA of the hip, treated with THA within 6 to 12 months of inclusion.

Exclusion criteria (both groups): Patients with a known history of symptomatic lung or heart disease or known symptoms of claustrophobia or unease using a mask and patients not understanding Danish language were excluded. Patients dependent on walking aid (and therefore unable to comply with the free living protocol) were excluded as well. Finally, for the post surgery group, patients with a scheduled reoperation of the hip or previous dislocation were excluded.

Twenty five were asked, 3 declined to participate and 1 was excluded due to known symptomatic lung disease and 1 due to known symptoms of claustrophobia. All 20 participants were able to complete the free living scenario.

All participants gave informed written consent and the conditions and methods of the study protocol was approved by the Ethical Committee, Region of Copenhagen, Denmark (Identifier; H-2-2010-47) and performed in accordance with the Helsinki Declaration of 1975, as revised in 2000 .

\section{Equipment}

The activity monitor:

A small multisensory activity monitor (Sensewear Pro3 armband (SWA)) was positioned over the triceps brachii muscle of the right arm at the midpoint between the acromion and olecranon processes (size; $85.3 \mathrm{~mm} \times 53.4 \mathrm{~mm} \times$ $19.5 \mathrm{~mm}$ ). The activity monitor collects physiological data from following sensors; a 2 axial accelerometer, a heat flux sensor, a skin temperature sensor, a near body ambient temperature sensor, and a galvanic skin response sensor. The activity monitor uses an onboard algorithm (InnerView TM Professional software version 5.1.0) fitted with anthropometric data from the participant (gender, age, height, and weight). The output is energy expenditure $(\mathrm{kcal} / \mathrm{min})$ calculated by an internal inaccessible algorithm.

\section{Criterion Method:}

Indirect calorimetry: For validation of the SWA armband a portable metabolic monitor (Cosmed model $\mathrm{K}_{4} \mathrm{~b}^{2}$ ) was worn during the protocol. The $\mathrm{K}_{4} \mathrm{~b}^{2}$ weighs $1.5 \mathrm{~kg}$ including a battery and is mounted on the chest with a simple harness. The $\mathrm{K}_{4} \mathrm{~b}^{2}$ has been shown valid in comparison to Douglas bag method [36]. Prior to the study 
the apparatus had been serviced by the manufacture and validated against Douglas Bag by the authors (Data not shown). Before each test, the monitor was calibrated in accordance with the manufactures instructions. Energy expenditure ( $\mathrm{kcal} / \mathrm{min}$ ) was calculated from the breath-bybreath oxygen use and carbon monoxide production.

\section{Study protocol}

A two hour protocol of 8 activities of daily living was designed. Activities imitate common activities of daily living expected for the patient/age group. Activities were: I) rest; 53 minutes (which includes all periods of rest in sitting and supine position), II) a simple warm-up program with steps and multi-planar movements; 9 minutes, III) sitting and walking between chairs; 4 minutes, IV) ascending and descending stairs; 4 minutes (4 steps, step height $15 \mathrm{~cm}$ ), V) walking; normal; 15 minutes (self-paced) and brisk walking; 10 minute, VI) jogging; 5 minutes (or brisk walking), VII) outdoor gardening; 10 minutes (raking), and VIII) indoor cleaning; 10 minutes (sweeping floor).

All activities were supervised and performed in a consecutive order following the protocol without time breaks or discontinuity of measurements. Participants were instructed to perform the activities within the intensities of their daily living. If an activity was impossible to perform due to pain or impairment of hip movements a lower intensity level was selected and the alteration was registered.

Subjects were fasting and refrained from smoking and drinking coffee 1 hour prior to testing to diminish possible influence on the basic energy expenditure. Before each assessment, the activity monitor was initialized and fitted to the patient according to the manufacturer's instruction. The data was downloaded in 1 minute epochs by software provided by the manufacturer (InnerView Professional Research Software Version 5.1.0).

The K4b2 was calibrated and mounted on the participant. For acclimatization the subjects rested seated 10 minutes prior to the protocol. To identify the time periods of the individual activities during the later data analysis both units (the SWA and the K4b2) and the time scheme of the protocol were synchronized by an electronic clock. The validation procedure including the initial calibration of units was performed by the principal author.

\section{Data analysis}

Bias was defined as the difference between the activity monitor and indirect calorimetry outcomes (kcal/min). Activity specific bias was analyzed for each activity separately (the 15 time intervals coded \#1-\#15). To diminish possible carry over effects between intervals due to $\mathrm{VO}_{2}$ latency, the first minute of each interval (\#1-\#15) was excluded from the later mean bias analysis of each activity and intervals of 2 minutes and less (interval \#3 and \#5) were regarded non-conclusive results. Mean bias of all 15 intervals (\#1-\#15) are presented.

\section{Statistical analysis}

Statistical analysis was carried out using functional data analysis [37]. This approach treats an entire curve of observations as a single datum rather than a collection of separate observations. In the present context each time dependent trajectory of the activity monitor and indirect calorimetry represents an observation. The techniques allow for a flexible characterization of the dynamics with minimal assumptions. In contrast, traditional methods such as linear mixed models that are based on the individual time points impose a parameterization on the functional form of the mean.

Specifically, we are interested in estimating the first two functional moments of the data. The functional mean leads to the definition of a time dependent bias function that varies freely over durations of the activities.

From the second order moments the functional variance processes [38] and the correlation coefficient were estimated [39] where the former characterize the internal stability of the activity monitor and indirect calorimetry.

The first step was to project the observed data into function space. We used a cubic b-spline basis with a knot placed at every minute and a data adaptive roughness penalty on the second derivative. The penalty parameter was estimated using the generalized cross-validation criterion [37].

A two-way functional ANOVA model showed no significant effect of surgical status, thus this factor was removed and the following results are based on pooled data.

The bias function was estimated as the functional mean of the pair-wise differences between the activity monitor and the indirect calorimetry curves with corresponding $95 \%$ confidence bands estimated by the method described by Cuevas et al. (2006) using the L2 norm as proximity measure [40].

The mean and relative biases of each interval (\#1-\#15) was calculated by a numeric quadrature rule over the corresponding intervals and the confidence intervals were based on a pair-wise re-sampling procedure.

Statistical analysis was carried out using $\mathrm{R}$ version 2.15 .2 (2012-10-26) “Trick or Treat" Copyright (C) 2012 The R Foundation for Statistical Computing ISBN 3-900051-07-0.

\section{Results}

Descriptive characteristics of subjects are shown in Table 1.

All participants completed the protocol and all activities were performed according to the protocol except during activity \#13 in which all participants declined to perform jogging due to self esteemed lack of physical capability. Brisk walking was performed instead. 
Table 1 Subject characteristics

\begin{tabular}{llll}
\hline & Total $(\mathbf{n}=\mathbf{2 0})$ & Female $(\mathbf{n}=\mathbf{8})$ & Male $(\mathbf{n}=\mathbf{1 2})$ \\
\hline Age (years) & $63.3 \pm 9.2$ & $67.1 \pm 8.6$ & $60.7 \pm 9.0$ \\
Weight $(\mathrm{kg})$ & $82.8 \pm 15.0$ & $73.4 \pm 11.2$ & $89.0 \pm 14.3$ \\
Height $(\mathrm{m})$ & $174.2 \pm 7.7$ & $167.8 \pm 5.2$ & $178.5 \pm 5.9$ \\
BMI $\left(\mathrm{kg} / \mathrm{m}^{2}\right)$ & $23.7 \pm 3.8$ & $21.9 \pm 3.3$ & $24.9 \pm 3.8$ \\
\hline
\end{tabular}

Data are $\bar{x} \pm$ SD.

Figure 1 illustrates the mean bias (difference) between the SWA and indirect calorimetry as a continuous time function with $95 \%$ confidence intervals. The bias is mainly significant positive (overestimation) with a fluctuant pattern that appears to follow transitions in activity mode.

The total energy expenditure was overestimated by $72 \%$ by the SWA during giving a significant average overestimation of $1.5 \mathrm{Kcal} / \mathrm{min}, 95 \% \mathrm{CI}(1.3,1.8)$ during all activities (Table 2).

During walking activities (\#8, \#11, \#13) overestimation ranged between $62 \%$ and $93 \%$. Significant underestimation $(-25 \%)$ was observed during ascending/descending stairs (\#6) while intervals dominated by upper body movement (\#9 and \#15) showed large overestimation of $170 \%$ and $119 \%$ for outdoor gardening and indoor cleaning, respectively (Table 2).

Figure 2 illustrates the variance processes of the two methods and demonstrates the SWA to be less stable during most activities except for periods of resting. The correlation coefficient between methods (all activities) was 0.94 .

\section{Discussion}

In the present study the SWA activity monitor was validated during simulated activities of daily living in a group of patients with hip osteoarthritis before or after THA by means of indirect calorimetry. The main findings were significant overestimations of energy expenditure by the activity monitor of up till 170\% during common activities of daily living. Bias and variance showed dependency on the type of activity performed.

The SWA has been used for estimation of PA in various patient groups including patients with hip and knee OA [5,14,19-23]; however, to our knowledge no previous studies have investigated the validity of the SWA or other activity monitors in patients with OA of the hip. The majority of validation studies of have been conducted in healthy adults $[12,30-34,41]$ of which two studies have reported the SWA as a valid tool for estimation of cumulated daily energy expenditure in comparison with doubly labeled water $[12,30]$. This contrasts with the majority of the activity specific protocols (using indirect calorimetry as criterion method) reporting the validity to be dependent of both the intensity and type of activity $[31,33,34,41,42]$. Direction of bias during walking activities may change according to inclination [34] and overestimation has been reported during exercise of the upper extremities [31]. This is in correspondence with the current findings of underestimation during stair climbing activities and overestimation during horizontal walking and in activities dominated with upper body movements. In healthy elderly numbers of validation studies are few and inconclusive in particular regarding the validity during activities $[11,24]$. In a study of resting energy expenditure in healthy

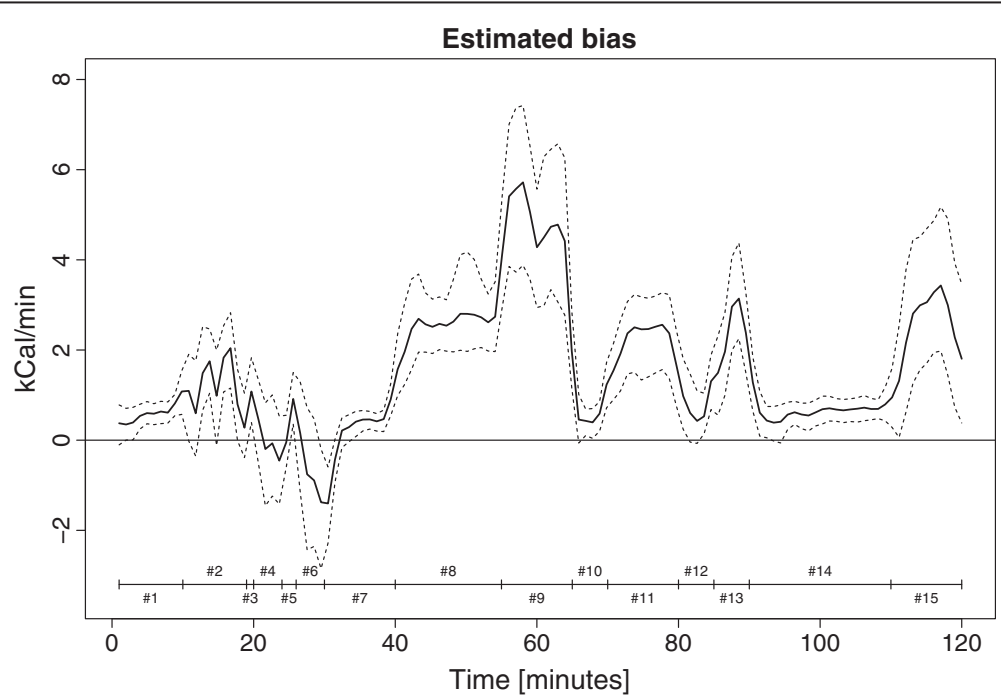

Figure 1 Bias between Sensewear Pro3 (SWA) estimates and indirect calorimetry (gold standard). Bias expressed as the mean difference with $95 \%$ confidence intervals. The horizontal line represents no difference between the methods. A positive value represents an overestimation of SWA. Coding \#1-\#15 represents intervals of steady state activity (see Table 2). 
Table 2 Activity types of the protocol with coding for intervals

\begin{tabular}{|c|c|c|c|c|c|c|}
\hline Activity type & $\begin{array}{l}\text { Length } \\
\text { (min) }\end{array}$ & Interval & $\begin{array}{l}\text { EE SWA } \\
\text { (kcal/min) }\end{array}$ & $\begin{array}{l}\text { EE IC } \\
\text { (kcal/min) }\end{array}$ & $\begin{array}{l}\text { Bias } \\
\text { (kcal/min) }\end{array}$ & Bias (\%) \\
\hline Total & 120 & & $3.7[3.4 ; 4.0]$ & $2.2[1.8 ; 2.6]$ & $1.54[1.3 ; 1.8]$ & $71.8[51.7 ; 92.8]$ \\
\hline Resting in chair & 10 & $\# 1$ & $1.5[1.4 ; 1.6]$ & $0.9[0.7 ; 1.1]$ & $0.6[0.5 ; 0.8]$ & $77.8[45.2 ; 117.5]$ \\
\hline Work out (steps and multi planar movements) & 9 & $\# 2$ & $4.2[3.8 ; 4.6]$ & $3.0[2.5 ; 3.5]$ & $1.2[0.7 ; 1.6]$ & $40.3[21.0 ; 60.8]$ \\
\hline Resting in chair ${ }^{2}$ & 1 & \#3 & $3.0[2.6 ; 3.4]$ & $2.3[1.9 ; 2.7]$ & $0.7[0.1 ; 1.2]$ & $29.6[5.4 ; 57.3]$ \\
\hline Sitting/standing and walking between 2 chairs & 4 & $\# 4$ & $3.6[3.1 ; 4.1]$ & $3.8[3.2 ; 4.4]$ & $-0.2[-0.8 ; 0.4]$ & $-4.7[-19.6 ; 10.5]$ \\
\hline Resting I chair ${ }^{2}$ & 2 & \#5 & $2.6[2.0 . ; 3.1]$ & $2.1[1.6 ; 2.5]$ & $0.5[0.1 ; 1.0]$ & $27.0[2.4 ; 59.3]$ \\
\hline Stair climbing (5 steps up/down) & 4 & \#6 & $3.1[2.7 ; 3.6]$ & $4.2[3.6 ; 4.9]$ & $-1.1[-1.8 ;-0.3]$ & $-24.8[-39.1 ;-7.6]$ \\
\hline Resting in a supine position & 10 & \#7 & $1.5[1.3 ; 1.6]$ & $1.0[0.8 ; 1.2]$ & $0.5[0.3 ; 0.7]$ & $53.1[25.6 ; 81.0]$ \\
\hline Walking normal speed (self paced) & 15 & \#8 & $5.8[5.1 ; 6.5]$ & $3.0[2.5 ; 3.5]$ & $2.8[2.3 ; 3.3]$ & $93.3[72.0 ; 119.1]$ \\
\hline Outdoor gardening (raking leaves) & 10 & \#9 & $7.0[6.1 ; 7.8]$ & $2.6[2.2 ; 3.1]$ & $4.4[3.8 ; 5.1]$ & $170.3[134.0 ; 211.4]$ \\
\hline Resting in chair & 5 & $\# 10$ & $1.8[1.6 ; 2.0]$ & $1.0[0.8 ; 1.3]$ & $0.8[0.5 ; 0.9]$ & $73.9[42.5 ; 105.7]$ \\
\hline Brisk walking & 10 & $\# 11$ & $5.7[5.2 ; 6.2]$ & $3.5[2.9 ; 4.1]$ & $2.2[1.7 ; 2.6]$ & $62.9[42.3 ; 87.2]$ \\
\hline Resting in chair & 5 & $\# 12$ & $2.1[1.8 ; 2.4]$ & $1.2[1.0 ; 1.5]$ & $0.9[0.6 ; 1.2]$ & $71.8[41.3 ; 107.7]$ \\
\hline Jogging/brisk walking & 5 & $\# 13$ & $6.1[5.2 ; 7.2]$ & $3.8[3.1 ; 4.5]$ & $2.3[1.8 ; 2.9]$ & $61.9[45.3 ; 81.9]$ \\
\hline Resting in chair & 20 & $\# 14$ & $1.4[1.3 ; 1.5]$ & $0.8[0.6 ; 1.0]$ & $0.7[0.5 ; 0.8]$ & $88.0[47.2 ; 136.2]$ \\
\hline Sweeping floor & 10 & $\# 15$ & $5.0[4.2 ; 5.8]$ & $2.3[1.9 ; 2.8]$ & $2.7[1.9 ; 3.5]$ & 119.4 [75.0; 172.1] \\
\hline
\end{tabular}

Mean values of energy expenditure (EE) measured by Sensewear Pro3 (SWA) and indirect calorimetry (IC) and absolute and relative bias between units ${ }^{1}$. Positive bias values indicate overestimation of SWA.

${ }^{1}$ Values are $\bar{x}$ with $95 \%$ confidence interval.

${ }^{2}$ Regarded as non conclusive due do short time period (see text).

elderly individuals (age (years); males 67.9 \pm 5.1 , females 69.2 \pm 5.1 ) Heiermann et al. (2011) found an overestimation (12-14\%) compared to indirect calorimetry [24] while Mackey et al. (2011) reported the SWA to be a valid tool for estimation of cumulated daily energy expenditure compared to doubly labeled water. Activity specific protocols in healthy elderly are currently lacking. In our population (age (years); $63.3 \pm 9.2$ ) we observed overestimation during rest and in the majority of activities (Table 2). Despite difficulty in comparison between studies the observed bias in the current study of hip OA patients appears larger than observations in healthy adults $[31,33,34,41,42]$. Meanwhile, recent validation studies in different elderly patient groups have indicated overestimation during various activities $[27,28]$. In elderly diabetic patients reported overestimations between $78 \%$ and $81 \%$ was reported

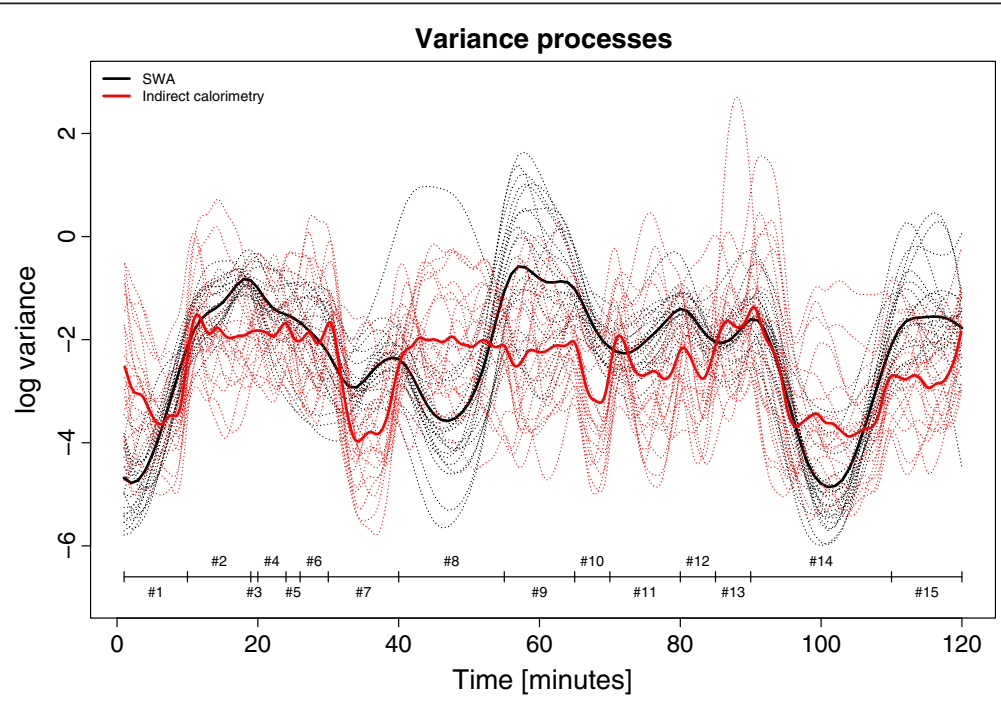

Figure 2 Functional variance processes of the Sensewear pro3 (SWA) and indirect calorimetry measurements showing the internal stability. Lower values indicate higher internal stability. Coding \#1-\#15 represents intervals of steady state activity (see Table 2). 
during horizontal walking, which is comparable with the current observations in hip OA patients [28]. In correspondence with observations in healthy adults [34] and the current observations (during stair climb), Machač et al. (2013) also reported underestimation during walking with inclination [28]. The present pronounced overestimation observed in household and garden activities involving upper body movements is likely to rely on the position of the monitor (accelerometer) on the upper arm.

Due to differences in criterion methods there are limitations concerning comparability of studies since the doubly labeled water method used for validation of daily energy expenditure $[11,12,30]$ does not allow for the activity specific validation attended in the current study design. Generally, studies in healthy adults have been performed at higher intensities compared to the current relatively low intensity protocol which may affect the observed validity. Additionally, the protocols differ largely between studies ranging from highly controlled activities (e.g. treadmill walking and RT-exercises) to various degrees of free living or simulated free living protocols. Physiological changes related to ageing has been suggested as a source to discrepancy between studies in elderly and younger adults [24], however the inaccessible inner algorithms deny further analysis of the individual contributions of the various physiological outputs of the SWA unit.

\section{Clinical implications}

The present findings raise concerns regarding the validity in patients with osteoarthritis of the hip, which is important for the application and interpretation of activity monitor estimates within the present patient group, as well as in comparison between patients and healthy subjects. Despite reported high correlation between the SWA and indirect calorimetry the large bias (overestimation) observed during a variety of common activities of daily living represents a major concern for the validity in patients with hip OA. Furthermore, since both bias and variance showed dependency of the type of activity performed, alteration in activities of daily living, as might be expected following surgery or non-surgical interventions may further compromise the validity in clinical use.

\section{Study limitations}

The relative low number of participants limits further subgroup analysis. The number of activities in the protocol is restricted which limits generalization of results regarding actual free living. As the overall intensity in the protocol was rather low and the protocol dictated a number of resting periods in between the activities, the bias of a possible carry-over effect (the physiological delay in obtaining steady state after a change in activity) was assumable low, supported by the observed stable bias during the long resting periods (\#1, \#7 and \#14) placed at the beginning, middle and end of the protocol (Figure 1). The intensity of the activities in the protocol makes the results applicable only to a sedentary lifestyle. However, since none of the participants complied to the highest intensity activity (jogging/running) we believe, the protocol reflected typical activities of daily living for the present patient group and a higher intensity protocol was redundant. The use of a walking aid may affect the outcome of activity monitors during walking activities and the exclusion of patients dependent on walking aid limits the generalization of the result regarding hip OA patients with severe functional impairments. Finally, it is a limitation that the outputs from the various sensors of the SWA are inaccessible for analysis regarding their individual contribution to the outcome and their contribution to the error of measurements since the monitor essentially is a "black box".

\section{Conclusion}

In patients with hip osteoarthritis the SWA activity monitor showed substantial bias (overestimation) during common activities of daily living, especially when involving the upper body. Despite a high correlation between the activity monitor and indirect calorimetry, the size and direction of bias and variance between methods varied between activities indicating limited validity of the estimations of physical activity in patients with hip osteoarthritis.

In perspectives, for future prospective studies in patients with hip OA (i.e. in cohort or interventional studies), further validation studies of activity monitors and accelometers are needed as this study emphasizes the importance of both patient and apparatus specific validation studies prior to a clinical application.

\section{Competing interests}

The authors declare that they have no competing interests.

\section{Authors' contributions}

$\mathrm{AH}$ : Conception and design, collection of data, analysis and interpretation of the data, drafting of the article, obtaining of funding. MR-L: Conception and design, analysis and interpretation of the data, technical and logistic support. AKJ: Analysis and interpretation of the data, statistical expertise, drafting the article. $\mathrm{RH}$ : Analysis and interpretation of the data, statistical expertise. LBA: Conception and design, analysis and interpretation of the data, critical revision of the article for important intellectual content. SO: Conception and design, analysis and interpretation of the data, critical revision of the article for important intellectual content. AH-L: Conception and design, analysis and interpretation of the data, drafting the article, Critical revision of the article for important intellectual content. All authors read and approved the final manuscript.

\section{Authors' information}

AH: MD, PhD student, Orthopedic Research Unit, Department of Orthopaedic Surgery and Traumatology, Odense University Hospital, Institute of Clinical Research, University of Southern Denmark.

ML: cand. scient, PhD student, Institute of Sport Sciences and Clinical Biomechanics, University of Southern Denmark.

AKJ: cand. polyt, Phd student, Department of biostatistics, Institute of Public Health, University of Southern Denmark.

$\mathrm{RH}$ : Associate professor, Department of biostatistics, Institute of Public Health, University of Southern Denmark. 
LBA: Professor, Dr.med, Chief of research, Institute of Sport Sciences and Clinical Biomechanics, RICH, Exercise Epidemiology, University of Southern Denmark.

SO: Professor, Dr.med, PhD, University of Southern Denmark, Chief of research, Orthopedic Research Unit, Department of Orthopaedic Surgery and Traumatology, Odense University Hospital, Institute of Clinical Research, Odense University Hospital.

AHL: Associate Professor, PhD, Orthopedic Research Unit, Department of Orthopaedics Surgery and Traumatology, Odense University Hospital.

\section{Acknowledgments}

The study was conducted with financial support by a research grant from the Danish Rheumatism Association (project no: R80-A1294). The authors certify that the grant sponsor has no involvement in study design, collection, analysis and interpretation of data; in the writing of the manuscript; and in the decision to submit the manuscript for publication.

\section{Declaration of contributions}

All authors have made substantial contributions to all three of the sections below:

(1) Conception and design of the study or acquisition of data, or analysis and interpretation of data.

(2) Drafting the article or revising it critically for important intellectual content.

(3) Final approval of the version to be submitted.

\section{Author details}

${ }^{1}$ Orthopedic Research Unit, Department of Orthopaedic Surgery and Traumatology, Odense University Hospital, Odense, Denmark. ${ }^{2}$ Institute of Clinical Research, University of Southern Denmark, Odense, Denmark. ${ }^{3}$ Department of Orthopaedic Surgery, Herlev University Hospital, Copenhagen, Denmark. ${ }^{4}$ Institute of Sport Sciences and Clinical Biomechanics, University of Southern Denmark, Odense, Denmark. ${ }^{5}$ Department of Biostatistics, Institute of Public Health, University of Southern Denmark, Odense, Denmark. ${ }^{6}$ Institute of Regional Health Research, University of Southern Denmark, Odense, Denmark. ${ }^{7}$ Department of Orthopaedic Surgery, Herlev University Hospital, Herlev Ringvej 75, 2750 Herlev, Denmark.

Received: 31 July 2013 Accepted: 11 February 2014 Published: 19 February 2014

\section{References}

1. Nuesch E, Dieppe P, Reichenbach S, Williams S, Iff S, Juni P: All cause and disease specific mortality in patients with knee or hip osteoarthritis: population based cohort study. BMJ 2011, 342:d1165.

2. Wallis JA, Webster KE, Levinger P, Taylor NF: What proportion of people with hip and knee osteoarthritis meet physical activity guidelines? A systematic review and meta-analysis. Osteoarthritis Cartilage 2013, 21:1648-1659.

3. Troiano RP, Berrigan D, Dodd KW, Mâsse LC, Tilert T, McDowell M: Physical activity in the United States measured by accelerometer. Med Sci Sports Exerc 2008, 40:181-188.

4. De Groot IB, Bussmann HJ, Stam HJ, Verhaar JA: Small increase of actual physical activity 6 months after total hip or knee arthroplasty. Clin Orthop Relat Res 2008, 466:2201-2208.

5. Holsgaard-Larsen A, Roos E: Objectively measured physical activity in patients with end stage knee or hip osteoarthritis. Eur J Phys Rehabil Med 2012, 48:1-9.

6. Farr JN, Going SB, Lohman TG, Rankin L, Kasle S, Cornett M, Cussler E: Physical activity levels in patients with early knee osteoarthritis measured by accelerometry. Arthritis Rheum 2008, 59:1229-1236.

7. Global Recommendations on Physical Activity for Health (WHO). Geneva; 2010.

8. McClave $\mathrm{S}$, Snider $\mathrm{H}$ : Use of indirect calorimetry in clinical nutrition. Nutr Clin Pract 1992, 7:207-221.

9. Haugen HA, Chan L-N, Li F: Indirect calorimetry: a practical guide for clinicians. Nutr Clin Pract 2007, 22:377-388.

10. Bassett DR, Rowlands A, Trost SG: Calibration and validation of wearable monitors. Med Sci Sports Exerc 2012, 44(1 Suppl 1):S32-S38.

11. Mackey DC, Manini TM, Schoeller DA, Koster A, Glynn NW, Goodpaster BH, Satterfield S, Newman AB, Harris TB, Cummings SR: Validation of an armband to measure daily energy expenditure in older adults. J Gerontol A Biol Sci Med Sci 2011, 66:1108-1113.

12. St-Onge M, Mignault D, Allison DB, Rabasa-Lhoret R: Evaluation of a portable device to measure daily energy expenditure in free-living adults. Am J Clin Nutr 2007, 85:742-749.

13. Cereda E, Turrini M, Ciapanna D, Marbello L, Pietrobelli A, Corradi E: Assessing energy expenditure in cancer patients: a pilot validation of a New wearable device. J Parenter Enter Nutr 2007, 31:502-507.

14. Waschki B, Kirsten A, Holz O, Müller K-C, Meyer T, Watz H, Magnussen H: Physical activity is the strongest predictor of all-cause mortality in patients with COPD: a prospective cohort study. Chest 2011 140:331-342.

15. Terwee CB, Bouwmeester W, van Elsland SL, de Vet HCW, Dekker J: Instruments to assess physical activity in patients with osteoarthritis of the hip or knee: a systematic review of measurement properties. Osteoarthritis Cartilage 2011, 19:620-633.

16. Watelain E, Dujardin F, Babier F, Dubois D, Allard P: Pelvic and lower limb compensatory actions of subjects in an early stage of hip osteoarthritis. Arch Phys Med Rehabil 2001, 82:1705-1711.

17. Reininga IHF, Stevens M, Wagenmakers R, Bulstra SK, Groothoff JW, Zijlstra W: Subjects with hip osteoarthritis show distinctive patterns of trunk movements during gait - a body-fixed-sensor based analysis. J Neuroeng Rehabil 2012, 9:3.

18. Chodzko-Zajko WJ, Proctor DN, Fiatarone Singh MA, Minson CT, Nigg CR, Salem GJ, Skinner JS: American College of Sports Medicine position stand. Exercise and physical activity for older adults. Med Sci Sports Exerc 2009, 41:1510-1530.

19. Scheers T, Philippaerts R, Lefevre J: Objectively-determined intensity- and domain-specific physical activity and sedentary behavior in relation to percent body fat. Clin Nutr 2013, 32:999-1006.

20. Donaire-Gonzalez D, Gimeno-Santos E, Balcells E, Rodríguez DA, Farrero E, de Batlle J, Benet M, Ferrer A, Barberà JA, Gea J, Rodriguez-Roisin R, Antó JM, Garcia-Aymerich J: Physical activity in COPD patients: patterns and bouts. Eur Respir J, 42:993-1002.

21. Leutwyler H, Hubbard EM, Jeste DV, Miller B, Vinogradov S: Associations of schizophrenia symptoms and neurocognition with physical activity in older adults with schizophrenia. Biol Res Nurs 2014, 16:23-30.

22. Gradaschi R, Camerini G, Carlini F, Sukkar S, Sopinaro N, Adami GF: Physical activity after surgically obtained weight loss: study with a SenseWear armband in subjects undergoing biliopancreatic diversion. Obes Surg 2013.

23. Cawthon PM, Blackwell TL, Cauley JA, Ensrud KE, Dam T-T, Harrison SL, Peters KW, Mackey DC: Objective assessment of activity, energy expenditure, and functional limitations in older men: the osteoporotic fractures in men study. J Gerontol A Biol Sci Med Sci 2013, 68:1518-1524.

24. Heiermann S, Khalaj Hedayati K, Müller MJ, Dittmar M: Accuracy of a portable multisensor body monitor for predicting resting energy expenditure in older people: a comparison with indirect calorimetry. Gerontology 2011, 57:473-479.

25. Papazoglou D, Augello G, Tagliaferri M, Savia G, Marzullo P, Maltezos E, Liuzzi A: Evaluation of a multisensor armband in estimating energy expenditure in obese individuals. Obesity (Silver Spring) 2006 14:2217-2223.

26. Dwyer TJ, Alison JA, McKeough ZJ, Elkins MR, Bye PTP: Evaluation of the SenseWear activity monitor during exercise in cystic fibrosis and in health. Respir Med 2009, 103:1511-1517.

27. Tierney M, Fraser A, Purtill H, Kennedy N: Study to determine the criterion validity of the SenseWear Armband as a measure of physical activity in people with rheumatoid arthritis. Arthritis Care Res (Hoboken) 2013, 65:888-895.

28. Machač S, Procházka M, Radvanský J, Slabý K: Validation of physical activity monitors in individuals with diabetes: energy expenditure estimation by the multisensor SenseWear Armband Pro3 and the step counter Omron $\mathrm{HJ}-720$ against indirect calorimetry during walking. Diabetes Technol Ther 2013, 15:413-418.

29. Brazeau A, Karelis A, Mignault D, Lacroix MJ, Rabasa-Lhoret R: Test-retest reliability of a portable monitor to assess energy expenditure. Appl Physiol Nutr Metab 2011, 36:339-343.

30. Johannsen DL, Calabro MA, Stewart J, Franke W, Rood JC, Welk GJ: Accuracy of armband monitors for measuring daily energy expenditure in healthy adults. Med Sci Sports Exerc 2010, 42:2134-2140. 
31. Jakicic JM, Marcus M, Gallagher KI, Randall C, Thomas E, Goss FL, Robertson RJ: Evaluation of the sensewear pro armband??? to assess energy expenditure during exercise. Med Sci Sport Exerc, 2004:897-904.

32. Berntsen S, Hageberg R, Aandstad A, Mowinckel P, Anderssen SA, Carlsen K-H Andersen LB: Validity of physical activity monitors in adults participating in free-living activities. Br J Sports Med 2010, 44:657-664.

33. Drenowatz C, Eisenmann JC: Validation of the SenseWear Armband at high intensity exercise. Eur J Appl Physiol 2011, 111:883-887.

34. Fruin ML, Rankin JW: Validity of a multi-sensor armband in estimating rest and exercise energy expenditure. Med Sci Sport Exerc 2004, 36:1063-1069.

35. Sanchez MM, Binkowitz BS: Guidelines for measurement validation in clinical trial design. J Biopharm Stat 1999, 9:417-438.

36. McLaughlin JE, King GA, Howley ET, Bassett DR, Ainsworth BE: Validation of the COSMED K4 b2 portable metabolic system. Int I Sports Med 2001, 22:280-284.

37. Ramsey J, Silverman BW: Functional Data Analysis. Springer; 2005.

38. Müller H-G, Stadtmüller U, Yao F: Functional variance processes. J Am Stat Assoc 2006, 101:1007-1018.

39. Yang W, Müller H: Functional singular component analysis. Society 2011, 73:303-324.

40. Cuevas A, Febrero M, Fraiman R: On the use of the bootstrap for estimating functions with functional data. Comput Stat Data Anal 2006, 51:1063-1074.

41. Benito PJ, Neiva C, González-Quijano PS, Cupeiro R, Morencos E, Peinado $A B$ : Validation of the SenseWear Armband in circuit resistance training with different loads. Eur J Appl Physiol 2011.

42. Koehler K, Braun H, de Marées M, Fusch G, Fusch C, Schaenzer W: Assessing energy expenditure in male endurance athletes: validity of the SenseWear Armband. Med Sci Sports Exerc 2011, 43:1328-1333.

doi:10.1186/1471-2474-15-43

Cite this article as: Hermann et al:: Low validity of the Sensewear Pro3 activity monitor compared to indirect calorimetry during simulated free living in patients with osteoarthritis of the hip. BMC Musculoskeletal Disorders 2014 15:43.

\section{Submit your next manuscript to BioMed Central and take full advantage of:}

- Convenient online submission

- Thorough peer review

- No space constraints or color figure charges

- Immediate publication on acceptance

- Inclusion in PubMed, CAS, Scopus and Google Scholar

- Research which is freely available for redistribution 